\title{
AN ADAPTIVE SYNTHESIS CALIBRATION METHOD FOR TIME- INTERLEAVED SAMPLING SYSTEMS
}

\author{
Huiqing Pan, Shulin Tian, Peng Ye
}

University of Electronic Science and Technology of China, College of Automation Engineering, Chengdu, Sichuan, 611731, China (凹 huiqing.pan@gmail.com,+86286183 1304,shulin@uestc.edu.cn,yepeng@uestc.edu.cn)

\begin{abstract}
In a parallel time-interleaved data sampling system, timing and amplitude mismatches of this structure degrade the performance of the whole ADC system. In this paper, an adaptive blind synthesis calibration algorithm is proposed, which could estimate the timing, gain and offset errors simultaneously, and calibrate automatically. With no need of an extra calibration signal and redesign, it could efficiently and dynamically track the changes of mismatches due to aging or temperature variation. A fractional delay filter is developed to adjust the timing mismatch, which simplifies the design and decreases the cost. Computer simulations are also included to demonstrate the effectiveness of the proposed method.
\end{abstract}

Keywords: time-interleaved sampling, channel mismatch, nonuniform, adaptive.

(C) 2010 Polish Academy of Sciences. All rights reserved

\section{Introduction}

Because of the limits of electronic component and manufacture technique, the timeinterleaved Analog-to-Digital Converter (TIADC) parallel sampling technique is the most effective method to meet high sampling rate and high resolution sampling requests [1]. Unfortunately, the performance of this system is degraded by the following effects: (1) The mismatching of amplitude among various channels. (2) The different phase delay of sampling synchronization circuits. The gain, offset, and timing mismatches lead to additional spurious components in the spectrum, and degrade the system Signal-to-Noise Ratio (SNR) [2]. So, digital post-processing methods are needed, when analog matching techniques are either too imprecise or too expensive.

Over the years, many methods have been proposed to compensate or reduce the errors effectively. For stationary mismatches, the generalized cross correlator [3] is a classical estimator, as well as the discrete-time Fourier-transform-based method [4] provides viable solutions to the delay estimation problem. An offset compensation method utilizing randomly chopped input signals is presented in [5]. And an identification of gain error is presented in [6]. When the mismatches are time-varying due to aging or temperature variation, adaptive tracking is necessary. An adaptive estimation method for timing mismatch can be found in [7], with constraining the finite impulse response (FIR) interpolating filter coefficients to be some functions of the timing mismatch. In [8] and [9], an adaptive compensation of gain and offset mismatches is introduced. However, these researches mainly aimed at one of the mismatches, and did not take the synthesis influence brought by all three mismatches into consideration. Also, in these approaches, estimation and calibration are separated, that makes the cost of processing unbearable for real-time systems.

In this paper, we develop a novel adaptive algorithm for estimating and calibrating the channel mismatches while the system is operating normally on an unknown signal, based on 
the control of synthesis cost function of three mismatches. Without an extra calibration signal and redesign, this method could adjust and track the mismatches in background. The use of fractional delay (FD) filter achieves the timing mismatch adjustment and simplifies the design.

\section{Adaptive synthesis calibration}

\subsection{Multi-channel system model}

Figure 1 illustrates the block diagram of a typical TIADC system. It consists of M parallel ADCs, which is called "channel" and works at the same sampling rate $f_{s} / M$ driven by a master sampling clock. After reorganization, the $M$ ADCs work equivalently as a single ADC operating at an $M$ times higher sampling rate.

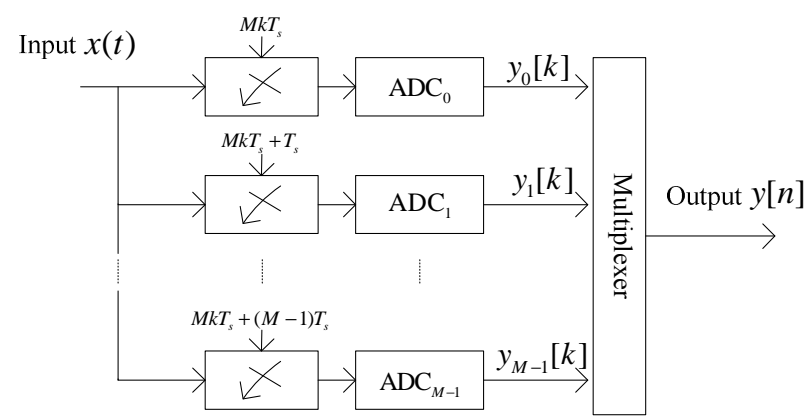

Fig. 1. Block diagram of a typical TIADC parallel system.

Unfortunately, because of the mismatching, each TIADC channel has gain, offset, and timing mismatch, namely, $g_{m}, o_{m}$, and $\Delta t_{m}$. The output of the $m$-th channel as illustrated in Fig. 2 is:

$$
y_{m}[k]=g_{m} x\left((k M+m) T_{s}+\Delta t_{m}\right)+o_{m}, k=0, \cdots, N-1, m=0, \cdots, M-1,
$$

where: $M$ denotes the number of channels, $x(t)$ represents the input signal, and $y_{m}[k]$ denotes the digital output signal of the $m$-th channel.

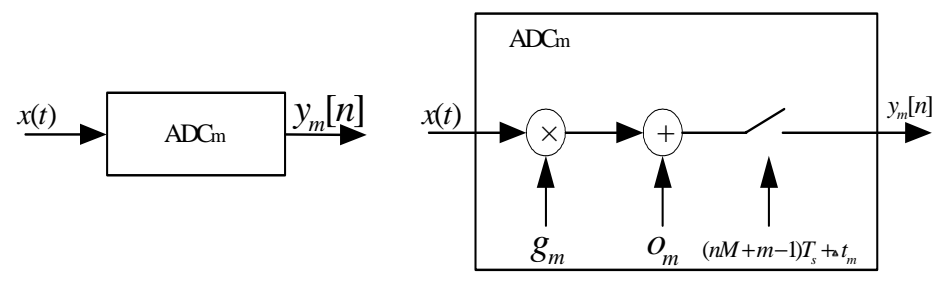

Fig. 2. Model of the $m$-th channel ADC.

The nonuniform signal degrades the system performance and has to be estimated and calibrated.

\subsection{Adaptive synthesis calibration algorithm}

When time-varying mismatches that were caused by aging or temperature variation are estimated, adaptive methods are generally used because of their general robustness. 
The adaptive filtering application is a recursive controller, which could modify its coefficients or control effect based on the variation of the signal parameters or the system state by constant measurements to attain the best possible performance. The distinguishing feature of adaptive filters is that they can modify their response to improve performance during operation without any intervention from the user. The adaptive system consists of the controllable module, controlled objects and adaptive controller. Let $x$ represent the input of the adaptive filter, $d$ be the desired response, $y$ denote the system output, the error signal be $e=d-y$; the goal is to adjust the system output to approximate the desired response as closely as possible according to a certain performance criterion by comparing its output to $d$ and to obtain a good estimation of the parameters.

In a TIADC parallel sampling system with band-limited input $x(t)$, the channel 0 is chosen as the reference channel, that is, $g_{0}=1, o_{0}=0, \Delta t_{0}=0$, its output $y_{0}[k]$ as the desire response, and the sampled signal of $m$-th channel $y_{m}[k]$ as the input of adaptive filtering system. According to optimal criterion, the synthesis calibration is to find the errors $g_{m}, o_{m}$, and $\Delta t_{m}$, making the mismatches between the $m$-th channel and the reference channel as small as possible through adjusting the system output $\hat{y}_{m 0}[k]$ to be closer to $y_{0}[k]$ based on the control of cost function.

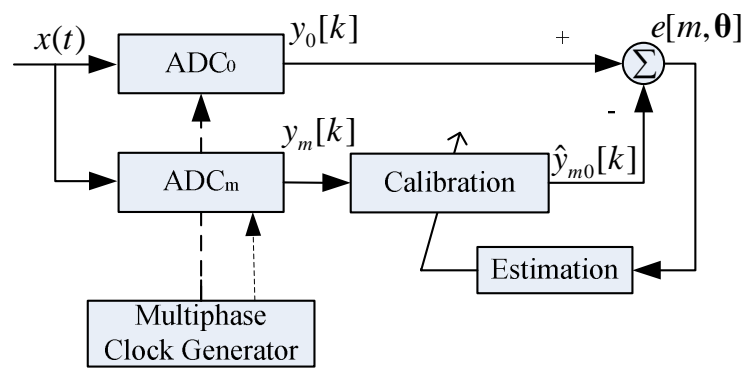

Fig. 3. Basic classes of adaptive filter configuration.

The error signal $e[m, \boldsymbol{\theta}]$ represents the difference between the estimated response $\hat{y}_{m 0}[k]$ and the desired response $y_{0}[k]$, that is:

$$
e[m, \boldsymbol{\theta}]=y_{0}[k]-\hat{y}_{m 0}[k],
$$

where: $\boldsymbol{\theta}$ denotes the vector of unknown parameters and $\hat{y}_{m 0}[k]$ represents the estimation of $y_{0}[k]$ using $y_{m}[k]$. Based on the channel module illustrated in Fig. 2 and Eq. (1), $\hat{y}_{m 0}[k]$ is calculated as:

$$
\hat{y}_{m 0}[k]=\frac{1}{\hat{g}_{m}} y_{m}\left[k,-\left(m+\hat{d}_{m}\right)\right]-\hat{o}_{m},
$$

where:

$$
d_{m}=\frac{\Delta t_{m}}{T_{s}}
$$

$y_{m}[k, d]$ represents the value sampled at time $(k M+m+d) T_{s} ; \hat{d}_{m}, \hat{g}_{m}, \hat{o}_{m}$ denote the estimation of $d_{m}, g_{m}, o_{m}$ respectively.

We design an optimum filter that estimates the unknown parameters $\boldsymbol{\theta}$ by minimizing the following cost function based on the Least Mean Square (LMS) criterion: 


$$
J_{L M S}=\min J=\min E\left\{e[m, \boldsymbol{\theta}] e[m, \boldsymbol{\theta}]^{T}\right\},
$$

where ${ }^{T}$ denotes the transpose. Eq. (5) is a three dimension Nonlinear Least Mean Squares (NLMS):

$$
J_{L M S}=f\left(d_{m}, g_{m}, o_{m}\right) .
$$

In order to solve the nonlinear optimization problem in Eq. (6), we use a relaxation-based algorithm [10] based on the LMS criterion, decompose it into three optimization problems, generate the proper control signal to update $d_{m}{ }^{(k)}, g_{m}{ }^{(k)}, o_{m}{ }^{(k)}$ separately, and minimize the cost function to realize the synthesis estimation.

Using the Steepest Descent algorithm, the parameters are then adjusted by directly updating as:

$$
\begin{gathered}
\boldsymbol{\theta}_{m}{ }^{(k+1)}=\boldsymbol{\theta}_{m}{ }^{(k)}-\mu_{\theta} \nabla J_{\boldsymbol{\theta}}{ }^{(k)}, \\
\nabla J_{\boldsymbol{\theta}}{ }^{(k)}=E\left\{2 e[m, \boldsymbol{\theta}]^{(k)} \nabla e[m, \boldsymbol{\theta}]^{(k)}\right\}, \\
\nabla e[m, \boldsymbol{\theta}]^{(k)}=\left[\frac{\partial e[m, \boldsymbol{\theta}]^{(k)}}{\partial d_{m}}, \frac{\partial e[m, \boldsymbol{\theta}]^{(k)}}{\partial g_{m}}, \frac{\partial e[m, \boldsymbol{\theta}]^{(k)}}{\partial o_{m}}\right]^{T},
\end{gathered}
$$

where, $\boldsymbol{\theta}_{m}{ }^{(k)}=\left[d_{m}{ }^{(k)}, g_{m}{ }^{\left({ }^{k}\right)}, o_{m}{ }^{\left({ }^{2}\right)}\right]^{T}$ represents the estimation parameter of the $k$-th step, $\mu_{\theta}$ is the step size.

Timing mismatch is one of the most significant mismatches, not only difficult to correct, but even more difficult to identify. Since timing mismatch arising in TIADC is typically small, and the analog reconstruction, in general, is computationally expensive or inaccurate, a Farrow structure filter [11] is employed to implement the evaluation and calibration of the timing mismatch.

$$
y_{m}[k, d]=y_{m}[k] \otimes h_{F D}(n, d),
$$

where $\otimes$ denotes conjugate, so Eq. (3) can be rewritten as:

$$
\hat{y}_{m 0}[k]=\frac{1}{\hat{g}_{m}} y_{m}[k] \otimes h_{F D}\left[n,-\left(m+\hat{d}_{m}\right)\right]-\hat{o}_{m} .
$$

The update function of the timing mismatch is:

$$
\begin{gathered}
d_{m}{ }^{(k+1)}=d_{m}{ }^{(k)}-\mu_{d} \nabla J_{d_{m}}(k), \\
\nabla J_{d_{m}}(k)=2 e[m, \boldsymbol{\theta}] \frac{\partial}{\partial d_{m}}\left\{\frac{1}{\hat{g}_{m}{ }^{(k)}} y_{m}[k] \otimes h_{F D}\left[n,-\left(m+\hat{d}_{m}{ }^{(k)}\right)\right]-\hat{o}_{m}{ }^{(k)}\right\} \\
=-2 e[m, \boldsymbol{\theta}] \frac{1}{\hat{g}_{m}{ }^{(k)}} y_{m}[k] \otimes h_{F D}^{\prime}\left[n,-\left(m+\hat{d}_{m}{ }^{(k)}\right)\right],
\end{gathered}
$$

where $\mu_{d}$ is the step size of the timing mismatch.

The reconstruction of signals with offset error and gain mismatch costs only little computational complexity. As the offset error is an additive noise to the input signal in time domain, the accumulator could be used to compensate. The update function is as follows:

$$
o_{m}{ }^{(k+1)}=o_{m}{ }^{(k)}-\mu_{o} \nabla J_{o_{m}}(k),
$$




$$
\begin{aligned}
\nabla J_{o_{m}}(k) & =2 e[m, \boldsymbol{\theta}] \frac{\partial}{\partial o_{m}}\left\{\frac{1}{\hat{g}_{m}{ }^{(k)}} y_{m}[k] \otimes h_{F D}\left[n,-\left(m+\hat{d}_{m}{ }^{(k)}\right)\right]-\hat{o}_{m}{ }^{(k)}\right\} \\
& =-2 e[m, \boldsymbol{\theta}],
\end{aligned}
$$

where $\mu_{o}$ is the step size of the offset.

The gain error is multiplicative noise to the input signal, which is calibrated by the timevarying multiplier. With the step size $\mu_{g}$, the update function of gain is:

$$
\begin{gathered}
g_{m}{ }^{(k+1)}=g_{m}{ }^{(k)}-\mu_{g} \nabla J_{g_{m}}(k), \\
\nabla J_{g_{m}}(k)=2 e[m, \boldsymbol{\theta}] \frac{\partial}{\partial g_{m}}\left\{\frac{1}{\hat{g}_{m}{ }^{(k)}} y_{m}[k] \otimes h_{F D}\left[n,-\left(m+\hat{d}_{m}{ }^{(k)}\right)\right]-\hat{o}_{m}{ }^{(k)}\right\} \\
=-2 e[m, \boldsymbol{\theta}]\left[\hat{g}_{m}{ }^{(k)}\right]^{-2} y_{m}[k] \otimes h_{F D}\left[n,-\left(m+\hat{d}_{m}{ }^{(k)}\right)\right] .
\end{gathered}
$$

\subsection{FD Filter}

FD filter is a discrete-time interpolator to approximate the desired frequency response $e^{-j \omega d}$. When the fractional delay $d$ is time-varying, it does not have to be redesigned. The basic idea of the FD filter is to approximate each coefficient in an FIR filter as a polynomial, and decompose into several parallel fixed FIR filters.

For instance, by expending each coefficient in the overall filter to a polynomial function of degree $P_{3}$ in $d$, the FIR is expressible as:

$$
\begin{aligned}
H_{F D}(z, d) & =\sum_{n=0}^{P_{2}-1} h(n, d) z^{-n} \approx \sum_{n=0}^{P_{2}-1}\left[\sum_{l=0}^{P_{3}-1} c_{l}(n) d^{l}\right] z^{-n} \\
& =\sum_{l=0}^{P_{3}-1}\left[\sum_{n=0}^{P_{2}-1} c_{l}(n) z^{-n}\right] d^{l}=\sum_{l=0}^{P_{3}-1} C_{l}(z) d^{l} .
\end{aligned}
$$

The Eq. (18) represents a classic filter structure that is called a Farrow structure. The desired fractional delay is achieved by directly multiplying the outputs of these filters with the value of the fractional delay parameter $d$. Fig. 4 shows a typical FD filter with Farrow structure:

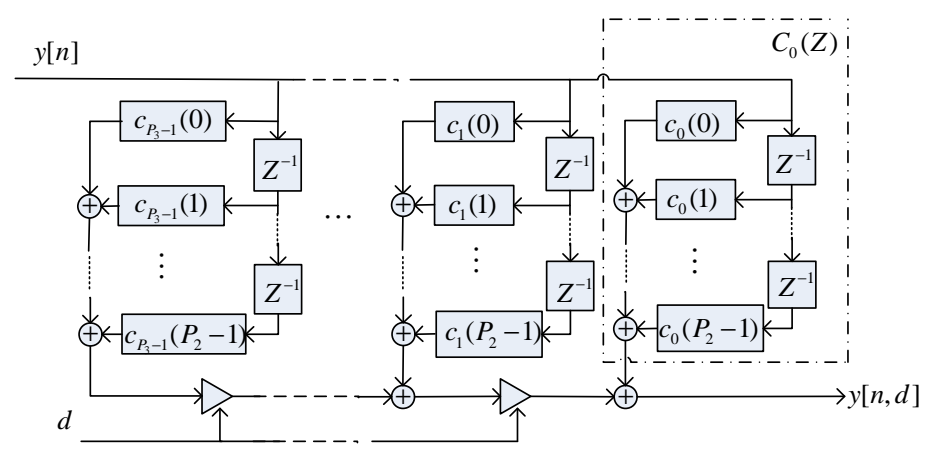

Fig. 4. Typical FD filter with Farrow structure. 
where, $P_{3}$ is the order of the polynomial and $c_{l}(n)$ is the coefficient for the $l$-st order term for the $n$-th value in the impulse response $h(n, d) . C_{l}(Z), l=0, \cdots, P_{3}-1$ are identified as FIR subfilters.

For the use of $\nabla J_{d_{m}}(k)$ in Eq. (18), the $\frac{\partial}{\partial d_{m}} H_{F D}(z, d)$ could be calculated as:

$$
\begin{aligned}
H_{F D}^{\prime}(n, d) & \stackrel{\Delta}{\partial d} H_{F D}(z, d) \\
& =\sum_{l=1}^{P_{3}-1} l c_{l}(n) d^{l-1} .
\end{aligned}
$$

The corresponding realization of the derivative of FD filter with a Farrow structure is shown in Fig. 5.

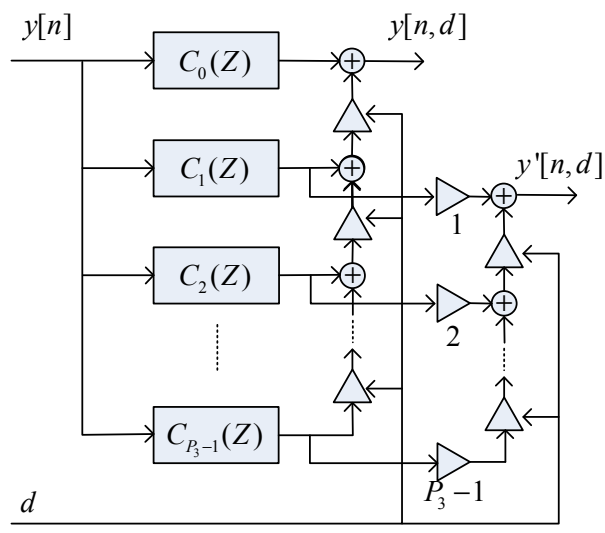

Fig. 5. A realization of the derivative of FD filter with a Farrow structure.

The FD filter can be designed using a constrained nonlinear optimization algorithm based on design criteria. For this purpose, the function fminimax from the optimization toolbox provided by Matlab [12] can be used. When using this function, we provide an objective function, that is, the error function minimized at the given frequency points as well as the gradient of the objective function with respect to the adjustable parameters. And the optimized coefficients of the FD filter with the frequency band $\Omega_{p}=[0,0.75 \pi]$ are as follows:

$$
\mathbf{C}=\left[\begin{array}{cccccccccccc}
-0.0103 & 0.0214 & -0.0449 & 0.0893 & -0.1870 & 0.6278 & 0.6278 & -0.1870 & 0.0893 & -0.0449 & 0.0214 & -0.0103 \\
0.0014 & -0.0016 & -0.0030 & 0.0136 & -0.0560 & 0.6200 & -0.6200 & 0.0560 & -0.0136 & 0.0030 & 0.0016 & -0.0014 \\
0.0080 & -0.0222 & 0.0443 & -0.0901 & 0.1886 & -0.1287 & -0.1287 & 0.1886 & -0.0901 & 0.0443 & -0.0222 & 0.0080 \\
-0.0044 & 0.0065 & -0.0016 & -0.0071 & 0.0485 & -0.1117 & 0.1117 & -0.0485 & 0.0071 & 0.0016 & -0.0065 & 0.0044
\end{array}\right]
$$

Fig. 6 illustrates the magnitude and phase delay responses for the optimized FD filter for $d=0,0.1,0.2, \ldots, 1$. 

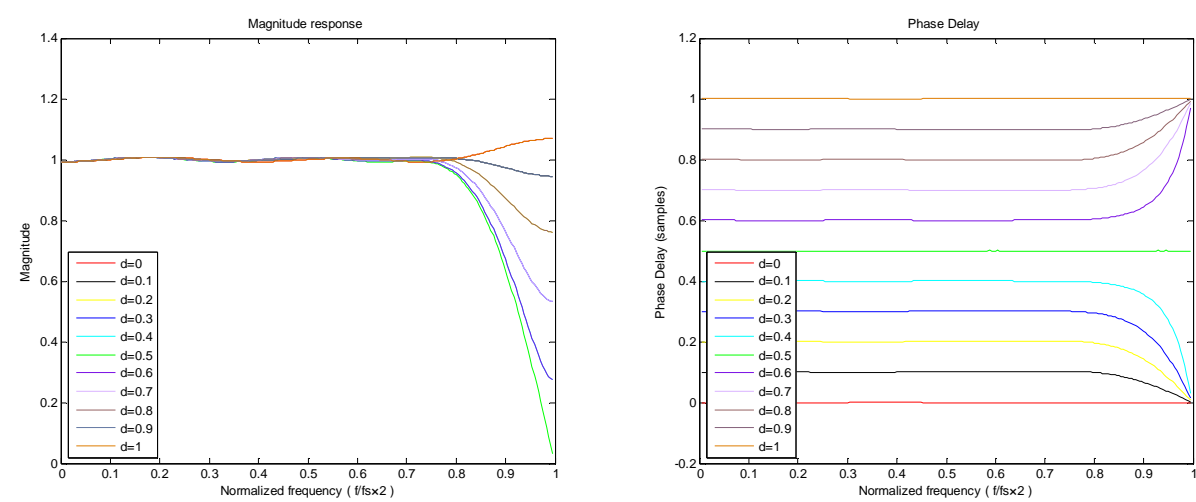

Fig. 6. Magnitude and phase delay response for FD filter for $\mathrm{d}=0,0.1,0.2, \ldots, 1$.

\subsection{Synthesis of calibration}

With the above analysis, the synthesis of the calibration algorithm is presented, and the Fig. 7.

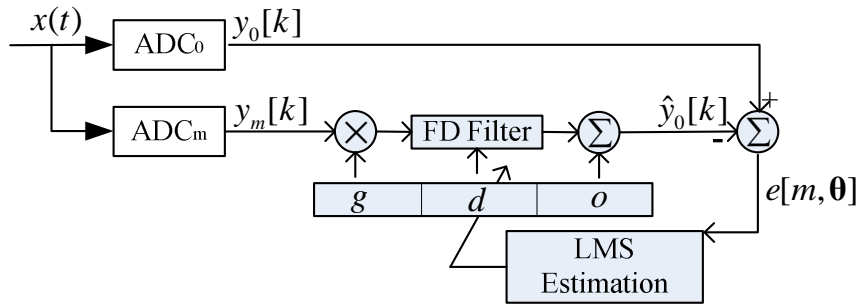

Fig. 7. Block diagram of adaptive synthesis calibration system.

- Step 1: select the initial conditions $d_{m}{ }^{(0)}, g_{m}{ }^{(0)}, o_{m}{ }^{(0)}$, and the optimal step sizes $\mu_{d}, \mu_{g}, \mu_{o}$;

- Step 2: sample the signal, obtain the data $y_{m}[k], m=0, \cdots, M-1$, and compute the deviation function $e[m, \boldsymbol{\theta}]$ according to Eq. (2);

- Step 3: iterate the update of $d_{m}{ }^{\left({ }^{+11}\right)}, g_{m}{ }^{(k+1)}, o_{m}{ }^{(k+1)}$ using Eq. (12), Eq. (16) and Eq. (14). If the difference between $J^{(k)}$ and $J^{(k-1)}$ is smaller than $10^{-3}$, then stop the adjustment, else repeat step 2;

- Step 4: refine the fractional delay parameter with $d$, then the calibration is finished.

Since the mismatching parameters' statistical properties of the TIADC system are unknown and change with time, the adaptive filter could automatically adjust its coefficients to compute instantly a "good" approximation at each time.

\section{Results and discussions}

Experiment 1 . The validity of the proposed adaptive synthesis calibration algorithm has been confirmed in a two-channel TIADC system with equivalent sampling rate at 500 MSPS supported by two 8Bit-250MSPS ADCs (Ideal_8_Bit.adc) [13]. Let the sampling system maintain certain nonuniform $d_{1}=0.16, g_{1}=1.09, o_{1}=0.025$, and apply the synthesis calibration algorithm to do verification.

A sine wave with frequency $f_{0}=10 \mathrm{MHz}$ is used as the test signal. The frequency response of the original output sequence before compensation is shown in Fig. 8. 

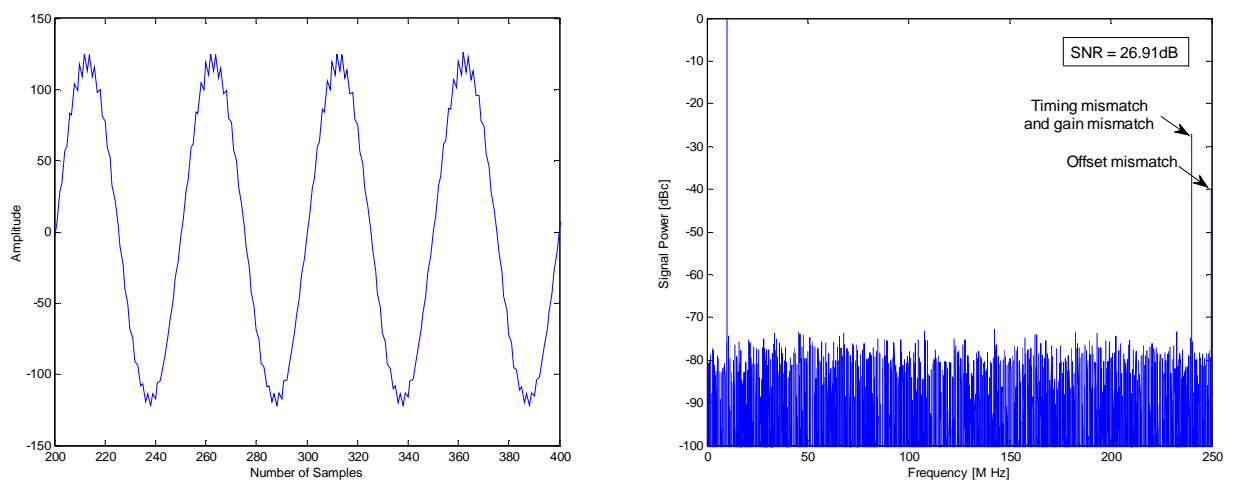

Fig. 8. Waveform and frequency spectrum of $10 \mathrm{MHz}$ sine wave captured by two-channel TIADC at $500 \mathrm{MSPS}$.

After adaptive synthesis calibration, at the step size $\mu_{d}=0.03, \mu_{g}=0.004, \mu_{o}=0.008$ which could obtain a good compromise between precision and time simulation, and the initial conditions $d_{1}{ }^{(0)}=o_{1}{ }^{(0)}=0, g_{1}{ }^{(0)}=1.1$, the convergences of the estimated timing, gain, offset mismatch with an increasing number of samples is shown in Fig. 9.

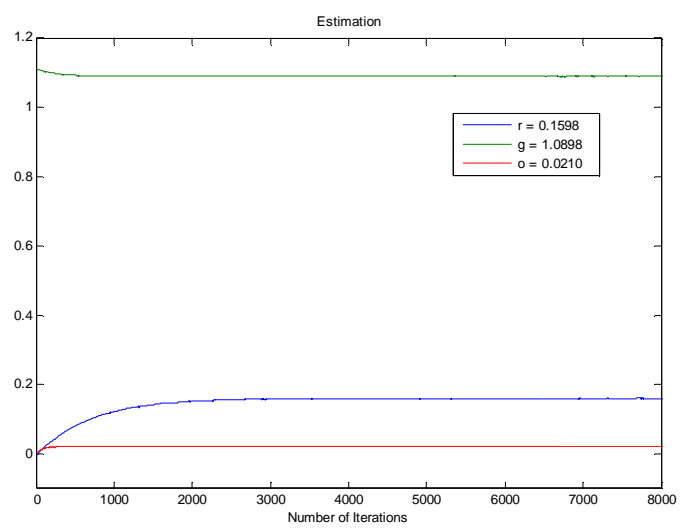

Fig. 9. The convergence behavior of the estimated timing, gain, offset mismatch.

The results show that the estimated mismatches are $\hat{d}_{1}=0.1598, \hat{g}_{1}=1.0898, \hat{o}_{1}=0.0210$, and the identification has effective accuracy. The gain and offset mismatch have converged to their correct value approximately after 300 samples, and timing mismatch has converged approximately after 2100 2500 samples.

Fig. 10 shows the waveform and frequency spectrum of the output signal after calibration.

As can be seen, the error spectra caused by mismatches have very little power. Now the calculated value of SNR is approximately $48.08 \mathrm{~dB}$ leading to an improvement of $21 \mathrm{~dB}$, the ENOB is increased by 7.69Bit-4.18Bit, and the SFDR is enhanced from $27.09 \mathrm{~dB}$ to $61.68 \mathrm{~dB}$. The performance of this TIADC system verifies the accurate mismatch estimation, and the additional spurious components in the spectrum have been reduced efficiently. 

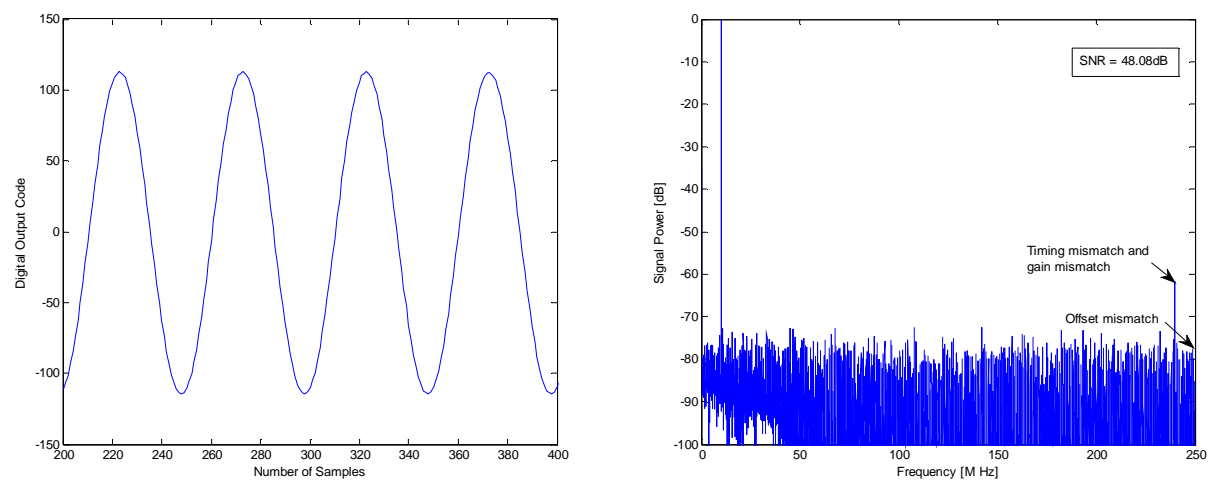

Fig. 10. Waveform and frequency spectrum of the calibrated output signal.

Experiment 2. In the same TIADC system with the same mismatches, the overall compensation performance has been evaluated by computing the signal-to-noise (SNR) for a different number of input frequencies.

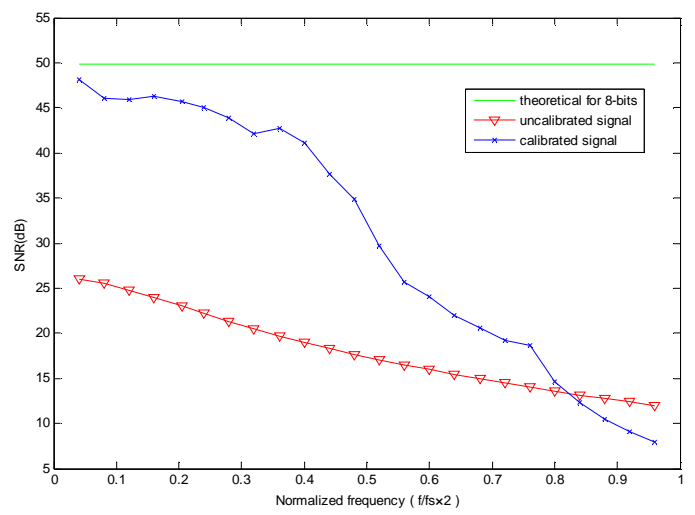

Fig. 11. SNR measurements for a sinusoidal input.

Fig. 11 shows the SNR for the calibrated and uncalibrated output signal. The top plot is the theoretical SNR of an 8 Bit ADC, the next and the bottom plot show the SNR of the signal with and without compensation respectively. Before calibration the maximum SNR is $27 \mathrm{~dB}$, and the calibration is necessary. We can observe that for very low frequency inputs, SNR improvement of the calibrated signal compared to the uncalibrated signal is evident. However, with the input frequency approaching the Nyquist frequency, the improvement becomes small. The SNR of the calibrated signal is superior to $40 \mathrm{~dB}$ for normalized frequency inputs up to approximately 0.4 . For very high-frequency inputs, the estimation does not converge effectively.

For most applications of the high speed Waveform Digitizer, the lowest band-width of the digitizer must be 5 times the centre frequency of the analyzed signal; it means that the ratio between the sampling rate of the TIADC and the frequency of the input is 0.2 0.4. So this synthesis calibration system works well. 


\section{Conclusions}

When using a parallel time-interleaved structure to realize high-speed sampling, the mismatches among the channels degrade the system's performance. In this paper, an adaptive blind synthesis calibration algorithm is presented, which could estimate and calibrate the timing, gain and offset errors automatically. With the use of a FD filter, it could compensate the timing error accurately with small cost. Without an extra calibration signal, this method could dynamically track the time-varying mismatches efficiently, and it especially suits a high-speed storage oscilloscope and other applications with high real-time and feasibility.

\section{Acknowledgements}

This work was supported by National Natural Science Foundation of China (grant No. 60772145 and grant No. 60827001).

\section{References}

[1] S.K. Mitra, A. Petraglia: "Analysis of mismatch effects among A/D converters in a time-interleaved waveform digitizer”. IEEE Trans. Instrum. Meas., vol. 40, no. 5, 1991, pp. 831-835.

[2] C. Vogel, D. Draxelmayr, G. Kubin: "Spectral shaping of timing mismatches in time-interleaved analog-todigital converters". ISCAS, 2005, pp. 1394-1397.

[3] C.H. Knapp, G.C. Carter: "The generalized correlation method for estimation of time delay". IEEE Trans Acoust Speech Signal Proces., vol. ASSP-24, 1976, pp. 320-327.

[4] Y.C. Jenq: "Digital spectra of nonuniformly sampled signals: a robust sampling time offset estimation algorithm for ultra high-speed waveform digitizers using interleaving". IEEE Trans. Instrum. Meas., vol. 39, no. 1, 1990, pp. 71-75.

[5] J.E. Eklund, F. Gustafsson: "Digital offset compensation of time-interleaved ADC using random chopper sampling”. ISCAS, vol. 3, 2000, pp. 447-450.

[6] S. Jamal, F. Daihong, N. Chang, P. Hurst, S. Lewis: "A 10-b 120-Msample/s time-interleaved analog-todigital converter with digital background calibration". IEEE J. Solid-State Circ., vol. 37, 2002, pp. 1618-1627.

[7] H.C. So, P.C. Ching, Y.T. Chan: "A new algorithm for explicit adaptation of time delay". IEEE Trans. Signal Proces., vol. 42, no. 7, 1994, pp. 1816-1820.

[8] D. Fu, K.C. Dyer, S.H. Lewis, P.J. Hurst: “A digital background calibration technique for time-interleaved analog-to-digital converters”. IEEE J. Solid-State Circ., vol. 33, 1998, pp. 1904-1911.

[9] T. Ndjountche, R. Unbehauen: "Adaptive calibration techniques for time-interleaved ADCs". Electronic Letter, vol. 37, 2001, pp. 412-414.

[10] L. Jian, W.R. Biao: “An efficient algorithm for time delay estimation”. IEEE Trans. Signal Proces., vol. 46, no. 8, 1998, pp. 2231-2235.

[11] Y.K. Juha, S. Tapio: "Multiplier-free polynomial-based FIR filters with an adjustable fractional delay". IEEE Trans. Circ., Syst., Signal Proces., vol. 25, no. 2, 2006, pp. 265-294.

[12] T. Coleman, M.A. Branch, A. Grace: Optimization Toolbox User's Guide Version 2, The MathWorks Inc., 1999, pp. 282-298.

[13] http://www.analog.com/en/analog-to-digital-converters/ad-converter/products/evaluationboardstools/CU_A DIsimADC_evaluation_tools/resources/fca.html.

[14]F.C. Alegria: "Study of the Random Noise Test of Analog-to-Digital Converters". Metrol. Meas. Syst., vol. XVI, no. 4, 2009, pp. 545-556. 